\title{
The Effects of Preoperative Alcohol, Tobacco, and Psychological Stress on Postoperative Complications: A Prospective Observational Study
}

Yoshinori Myoga ( $\nabla$ wakasakura2001@yahoo.co.jp )

Kitakyushu Municipal Medical Center

Haruhiko Manabe

Kitakyushu Municipal Medical Center

Yoneatsu Osaki

Tottori University

\section{Research Article}

Keywords: Alcohol, mental stress, postoperative complications, psychological stress, tobacco

Posted Date: September 27th, 2021

DOI: https://doi.org/10.21203/rs.3.rs-338240/v1

License: (c) This work is licensed under a Creative Commons Attribution 4.0 International License. Read Full License 


\section{Abstract}

Background: Postoperative complications occur frequently, despite progress in anesthetic pharmacology and surgical techniques. Although habits, such as alcohol and tobacco use, and mental health have been studied individually as modifying factors, few studies have examined the relationship between multiple lifestyle choices and postoperative complications in patients undergoing surgery. Hence, this study aimed to investigate the associations between unhealthy lifestyle choices and postoperative complications.

Methods: We included 730 patients who underwent surgery in our department between March 2015 and April 2016. Participants completed preoperative questionnaires, including the Alcohol Use Disorders Identification Test, Fagerström Test for Nicotine Dependence, and tests for psychological stress (6-item Kessler Psychological Distress Scale; Hospital Anxiety and Depression Scale). Multivariable logistic analysis was used to analyze the association of preoperative drug dependence and psychological stress with postoperative complications.

Results: Of the 721 cases analyzed, 461 (64\%) were women. The median age of patients was 62 years (interquartile range: $48-71$ ). At the time of surgical decision-making, 429 out of 710 respondents $(60 \%)$ had a drinking habit, and 144 out of 693 respondents (21\%) had a smoking habit during the preceding year. Seventy-nine patients had developed complications. Multivariable analysis revealed that old age $(p=0.020)$, psychological stress $(p=0.041)$, and longer anesthesia time $(p<0.001)$ were significantly associated with postoperative complications. Drinking or smoking variables were not associated with postoperative complications.

Conclusions: Preoperative psychological stress, as evaluated with the 6-item Kessler Psychological Distress Scale, is associated with the risk of postoperative complications.

\section{Background}

Despite advances in surgical procedures and anesthetic pharmacology, postoperative mortality rates of $0.5-4.0 \%$, mostly attributed to postoperative complications, have been reported in international multicenter cohort studies $[1,2]$. Postoperative complications affect $16.8 \%$ of elective surgeries, and between 30 and $60 \%$ in major abdominal surgeries [1, 3]; the patient's associated risk factors are typically old age, multiple comorbidities, frail, and unhealthy lifestyle and behavioral factors [4]. Although lifestyle factors, such as alcohol and tobacco use, reduce psychophysiological resilience and delay postoperative early recovery, those factors, including psychological stress, can be mitigated preoperatively [5-8]. By doing so, we can enhance the physiological and psychological resilience of patients and reduce postoperative complications [9].

Unhealthy lifestyle factors frequently cluster in the same individual and hence multimodal interventions for modifiable risk factors are warranted to enhance resilience in the time-pressured preoperative window $[10,11]$. However, lifestyle risks on surgical outcomes are often studied separately, to our knowledge, the association of multiple lifestyle risk factors with postoperative complications has not been studied in-depth in the surgical patient population [5-8]. Predicting the susceptibility of individuals to postoperative complications and assessing the impact of smoking, alcohol, psychological stress, and their interactions on postoperative complications is important to prevent postoperative mortality.

This study assessed the effects of preoperative alcohol and tobacco use, and psychological stress on postoperative complications. 


\section{Methods}

\section{Study Sample}

Following the Declaration of Helsinki, this study was conducted after obtaining approval from the Ethics Committee of the Kitakyushu Municipal Medical Center and Faculty of Medicine, Tottori University (Approval No.: 2662). The study participants were adult patients who underwent planned non-cardiac surgery with intubation under general anesthesia at the Kitakyushu Municipal Medical Center between March 2015 and April 2016.

A total of 730 participants completed the questionnaires to evaluate preoperative psychological stress, drinking/smoking habits, and alcohol/nicotine dependence. Inclusion criteria included patients between categories I and III in the American Society of Anesthesiologists physical status classification (ASA-PS), who were at least 20 years of age. Exclusion criteria included the inability to complete to the questionnaire due to dementia or dysgraphia, as judged by the anesthesiologists.

\section{Procedure}

Questionnaires were given to patients at the preoperative consultation (one to three days before surgery). Anesthesiologists ( $\mathrm{YM}$ and $\mathrm{HM}$ ) provided a verbal explanation of the study using the patient's manual, including the study details, such as purpose, methods, the right to refuse participation, and contact information for questions and complaints related to the study (Opt-in and Opt-out). Patients provided completed and signed questionnaires to attending anesthesiologists, implying consent for the study.

We analyzed how drinking/smoking habits, the degree of dependence, and the degree of psychological stress were related to postoperative complications within 30 days of surgery. The patients' background, surgical, and anesthesia data were obtained from the electronic medical records and preoperative questionnaires. We also investigated the duration of hospitalization and in-hospital death as secondary outcomes. Postoperative complications included those that met the Japan Clinical Oncology Group (JCOG) postoperative complications criteria, as well as those that required additional treatment, such as hyperactive delirium, asthma, and catheter infections.

\section{Measurements (covariates)}

We assessed factors known to affect postoperative complications to include as covariates, including age, sex, body mass index (BMI), ASA-PS (as determined by attending anesthesiologists), Preoperative use of psychotropics (hypnotics, anxiolytics, and antidepressants), anesthesia time/surgical time, and the Surgical Apgar score (sAs, collected from the electronic anesthesia records by the first author). Details of each evaluation tool are outlined below.

\section{Alcohol screening in the year prior to surgery}

The Alcohol Use Disorder Identification Test (AUDIT) is a self-administered screening test that was developed by the WHO in the 1980 s to evaluate alcohol consumption, dependence, and problematic drinking [12]. The AUDIT 
includes 10 items and can be used across various ethnicities and cultures. Respondents are asked to answer 10 questions regarding alcohol use in the preceding year; items 1-3 assess the amount and frequency of alcohol consumption, items 4-6 assess the degree of dependence, and items 7-10 assess problematic drinking, with a total score of $0-40$ points. The cutoff value for problematic drinking is 8 points or higher.

\section{Tobacco screening in the year prior to surgery}

The Fagerström Test for Nicotine Dependence (FTND) is a modified version of the Fagerström Tolerance Questionnaire and was developed by Heatherton et al. to assess the need for nicotine replacement therapy in the treatment of withdrawal symptoms among smokers who are trying to quit [13]. This simplified test contains six items that assess tobacco consumption and the degree of dependence. Each item has a varying degree of importance (points), and scores are summed to yield a total score of 10 . The degree of dependence is categorized as follows: Low dependence (0-2 points), medium dependence (3-6 points), and high dependence (7-10 points) [14]. The cutoff for nicotine dependence was 3 points or higher.

\section{Mental health screening 30 days prior to surgery}

In the medical field, the Hospital Anxiety and Depression Scale (HADS) is a well-established self-administered 14item scale that has two subscales that assess anxiety (7 items) and depression (7 items). The HADS was developed by Zigmond and Snaith in 1983 to evaluate the degree of anxiety and depression in non-psychiatric hospitals and clinics [15]. Items related to physical symptoms, such as dizziness, headache, and low appetite were not included as they are not depression-specific symptoms. Respondents rated each HADS item on a 4-point scale (0-3 points), whereby a score of 0 is the most positive, and a score of 3 is the most negative. A higher HADS score represents a greater risk of anxiety and depressive disorders. A score of 11-19 points indicates adjustment disorder, and a score of 20 or more indicates major depressive disorder [16].

Meanwhile, the 6-item Kessler Psychological Distress Scale (K6) is a simplified version of the 10-item version of the scale and evaluates nonspecific psychological stress in workplaces and the field of epidemiology [17], hence K6 can assess the overall psychological stress, such as mood and anxiety disorders, but not depression. Arnaud et al. reported that the $\mathrm{K} 6$ is a convenient tool with a reliability that is equivalent to that of the $\mathrm{K} 10$ [18]. In this study, we used the Japanese version of the $\mathrm{K} 6$, which includes items 2, 4, 5, 8, 9, and 10 of the K10. Each $\mathrm{K} 6$ item is scored on a scale of 1 to 5 (1: never; 2: rarely; 3: some of the time; 4: most of the time; 5 : all the time), with a total score of 0-24 points. We defined that a score of 8 points or higher was considered as indicative of psychological stress [19].

\section{The preoperative severity of patient comorbidity (ASA-PS)}

The ASA-PS was developed by Meyer Saklad in 1941 [20]. The goal of the ASA-PS classification is to assess the overall health status and comorbidities of preoperative patients. Items are graded on a 6-point scale crudely, whereby a higher score indicates a higher risk of perioperative complications, 30-day readmissions, and longer hospitalization [20,21]. This is a subjective classification that is completed by the evaluator (an attending anesthesiologist) and does not consider surgical invasiveness, the method of anesthesia, or other intraoperative 
factors. Given that this is only a preoperative evaluation of a patient, we also used the sAs as an objective measure of intraoperative invasiveness in this study.

\section{The intraoperative surgical and anesthetic invasiveness (sAs)}

Following the Apgar score in obstetrics, Gawande et al. proposed the sAs in 2007, which has a maximum score of 10 points [22]. This total score is calculated by quantifying and scoring three parameters from intraoperative anesthesia records, including lowest mean blood pressure, lowest heart rate, and amount of blood loss. The amount of blood loss is an indicator of surgical complexity and the surgeon's performance, and changes in heart rate and blood pressure reflect the patient's physiological state and the appropriateness of anesthetic management. This scoring system effectively predicts the postoperative 30-day mortality rate and postoperative complications, for both general surgery and vascular surgery [23]. A maximum sAs (10 points) indicates the most appropriate intraoperative management.

\section{Assessment of Postoperative Complications}

The main outcome of this study was the occurrence of 30-day postoperative complications that required additional treatment or extended hospitalization. All complications were defined by the adverse event (AE) terms from the JCOG postoperative complications criteria. Those that were not included in the AE terms were classified as "others" (e.g., delirium, bronchial asthma, and catheter infections, among others).

\section{JCOG postoperative complications criteria version 2}

This measure is based on the original article describing the Clavien-Dindo classification of surgical complications (2004) [24], which has been frequently used to objectively assess procedural skills and 72 postoperative complications (73 items including "Others") [25]. The AE terms and each of their grading details were standardized from the Clavien-Dindo classification, with the approval of the JCOG operational committee. Version 2 (2013) is the most recent. Furthermore, AE terms such as pneumonia, ileus, anastomotic leak, wound infection, and postoperative hemorrhage were graded, from grade I to grade $\mathrm{V}$ (death). This study did not take the severity of complications into consideration.

\section{Statistical Analysis}

For statistical testing of medians, the Mann-Whitney $U$ test was used for the analysis for the comparison of patients with and without postoperative complications. For statistical testing of proportions, the chi-squared test was used to test the difference in proportion for the comparison of patients with and without postoperative complications. When the expected numbers for the chi-squared test were small, we used a Fisher's exact test. We conducted multiple logistic regression analysis. The dependent variable was postoperative complications, and independent variables were age, sex, BMI, ASA-PS, Preoperative use of psychotropics, sAs, anesthesia time, drinking and smoking habits (AUDIT, FTND), HADS, and K6. Model 1 included improved drinking/smoking habits after surgical decision-making as a covariate, and Model 2 included the degree of alcohol/nicotine dependence at the time of surgical decision-making as a covariate. A male and female subset of Model 2 (Model 3 and 4, 
respectively) was also created. Taking multicollinearity into consideration, for a correlation of 0.4 and above, one of the two variables was removed. We used the c-statistic (area under the curve [AUC]) and accuracy as indicators of the model's predictive precision. JMP version 12 (from SAS) was used for modeling, with a significance cutoff of $p<0.05$.

We assigned zero points to missing items in the AUDIT, FTND, K6, and HADS if more than $75 \%$ of all questions had been answered. The questionnaires with the following missing responses were excluded from the analysis: AUDIT responses with items 1-3 missing (questions related to the amount and frequency of drinking), FTND responses with item 4 missing (number of cigarettes smoked per day), and responses with $25 \%$ or more missing items ( 3 or more items in the AUDIT, 2 or more items in the FTND and K6, and 4 or more items in the HADS).

\section{Results}

A final total of 721 patients provided the questionnaires and were included in the analysis. Table 1 shows the type of surgery and the incidence of postoperative complications within 30 days of surgery. Of the $461(64 \%)$ women, $208(29 \%)$ were breast surgery cases. The rate of postoperative complication development was $11 \%$ in 79 cases, which included anastomotic leak/peritonitis $(n=11)$, wound infection/dehiscence $(n=8)$, postoperative hemorrhage $(n=5)$, and myocardial infarction $(n=1)$. The duration of postoperative hospitalization was longer in patients with postoperative complications than those without complications $(p<0.001)$. 
Table 1. Details of the surgery and postoperative complications

Type of surgery

n (\%)

Breast surgery

$209(29.0)$

Gastrointestinal surgery

$129(17.9)$

Orthopedic surgery

$96(13.3)$

Otolaryngology surgery

$66(9.2)$

Abdominal surgery for gynecology

$51(7.1)$

Cholecystectomy, staging laparoscopy, stoma construction/closure

$33(4.5)$

Thoracic surgery

$29(4.0)$

Thyroid/parathyroid surgery

$27(3.7)$

Hepatic surgery

$23(3.2)$

Abdominal surgery for urology

$19(2.6)$

Pancreatic surgery

$17(2.4)$

Transvaginal/urethral surgery

$8(1.1)$

Craniotomy

$3(0.4)$

Hernia repair

2 (0.3)

Others (minor surgery)

9 (1.3)

(continued)

Postoperative complications

n (\%)

Anastomotic leak/Peritonitis

$11(1.5)$

Pancreatic fistula/Gallbladder fistula

$9(1.2)$

Wound infection/dehiscence

$8(1.1)$

Intra-abdominal abscess

$8(1.1)$

Ileus/Gastroesophageal reflux disease

$6(0.8)$

Postoperative hemorrhage

$5(0.7)$

Atrial Fibrillation

$4(0.6)$

Pneumonia/Atelectasis

$3(0.4)$

Anastomotic stenosis

$3(0.4)$

Pulmonary fistula/Pleural effusion

$2(0.3)$

Enterovesical fistula/Rectalvaginal fistula

$2(0.3)$

Ischemic heart disease

$1(0.1)$

Implant infection

$1(0.1)$ 
Other

Hyperactive delirium

(continued).

Bronchial asthma

Infection of Infusion line

Herpes zoster, influenza, pustulosis, diverticulitis

$4(0.6)$

In-hospital death (137 days after surgery)

In-hospital death after readmission

1

Reoperation within 30 days of surgery

12

Readmission

4

Length of stay after surgery (days)

$9(6-13)$

Details of postoperative complications in 721 patients within 30 days of surgery. A total of 80 complications were found in 79 out of 721 patients (11\%). One patient experienced both atrial fibrillation and pleural effusion.

At the time of the surgical decision, 429 out of 710 respondents (60\%) had a drinking habit, and 144 out of 693 respondents $(21 \%)$ had a smoking habit in the preceding year. Within those with either drinking or smoking habits in the preceding year, $62 \%$ and $71 \%$ of patients, respectively, had either reduced or quit their habits by the day before surgery (Table 2). We applied univariable analysis of each factor against sex and postoperative complications. The duration of surgery and anesthesia were significantly longer in patients with postoperative complications $(p<0.001)$.

Table 3 shows the sex- and age-adjusted odds ratio (OR) and 95\% confidence interval (Cl) of each factor. Anesthesia time (hours) (OR: 1.53 [Cl: 1.37-1.73], p < 0.001), sAs (OR: 0.82 [Cl: 0.70-0.97], p = 0.019), and K6 score (OR: 1.07 [Cl: 1.00-1.13], $p=0.040$ ) were significant risk factors associated with the development of postoperative complications. Preoperative use of psychotropics were not associated with postoperative complications. 
Table 2

Association of postoperative complications and patient characteristics by sex

\begin{tabular}{|c|c|c|c|c|c|c|c|}
\hline & \multirow[t]{3}{*}{ All (721) } & \multicolumn{3}{|l|}{ Male (260) } & \multicolumn{3}{|l|}{ Female (461) } \\
\hline & & \multicolumn{3}{|c|}{$\begin{array}{l}\text { Postoperative } \\
\text { complications }\end{array}$} & \multicolumn{3}{|l|}{$\begin{array}{l}\text { Postoperative } \\
\text { complications }\end{array}$} \\
\hline & & No (215) & $\begin{array}{l}\text { Yes } \\
(45)\end{array}$ & p value & No (427) & $\begin{array}{l}\text { Yes } \\
(34)\end{array}$ & $p$ value \\
\hline Age (years) & $62(48-71)$ & $65(52-72)$ & $\begin{array}{l}66(59- \\
72)\end{array}$ & 0.27 & $57(47-69)$ & $\begin{array}{l}67(62- \\
73)\end{array}$ & $0.001 *$ \\
\hline $\mathrm{BMI}\left(\mathrm{kg} / \mathrm{m}^{2}\right)$ & $23(21-25)$ & $24(22-27)$ & $\begin{array}{l}23(22- \\
25)\end{array}$ & 0.18 & $22(20-25)$ & $\begin{array}{l}22(19- \\
24)\end{array}$ & 0.39 \\
\hline ASA-PS I/II/III & $212 / 483 / 26$ & $30 / 175 / 10$ & $2 / 41 / 2$ & 0.21 & $172 / 242 / 13$ & $8 / 25 / 1$ & 0.15 \\
\hline $\begin{array}{l}\text { Preoperative use } \\
\text { of psychotropics }\end{array}$ & $\begin{array}{l}85 / 693 \\
(12 \%)\end{array}$ & $\begin{array}{l}23 / 206 \\
(11 \%)\end{array}$ & $\begin{array}{l}5 / 45 \\
(11 \%)\end{array}$ & 1.00 & $\begin{array}{l}51 / 409 \\
(12 \%)\end{array}$ & $\begin{array}{l}6 / 33 \\
(18 \%)\end{array}$ & 0.41 \\
\hline $\begin{array}{l}\text { Surgical time } \\
\text { (minutes) }\end{array}$ & $\begin{array}{l}125(81- \\
201)\end{array}$ & $\begin{array}{l}104(66- \\
227)\end{array}$ & $\begin{array}{l}262 \\
(186- \\
344)\end{array}$ & $p<0.001^{*}$ & $\begin{array}{l}118(85- \\
161)\end{array}$ & $\begin{array}{l}196 \\
(131- \\
294)\end{array}$ & $p<0.001 *$ \\
\hline $\begin{array}{l}\text { Anesthesia time } \\
\text { (minutes) }\end{array}$ & $\begin{array}{l}176(134- \\
254)\end{array}$ & $\begin{array}{l}173(107- \\
282)\end{array}$ & $\begin{array}{l}305 \\
(236- \\
443)\end{array}$ & $p<0.001 *$ & $\begin{array}{l}168(135- \\
218)\end{array}$ & $\begin{array}{l}239 \\
(179- \\
359)\end{array}$ & $p<0.001$ * \\
\hline sAs & $8(7-9)$ & $8(7-8)$ & $7(6-8)$ & $0.039 *$ & $8(7-9)$ & $7(7-8)$ & 0.057 \\
\hline Drinking \lifetime $\mathbb{Z}$ & $\begin{array}{l}514 / 710 \\
(72 \%)\end{array}$ & $\begin{array}{l}185 / 213 \\
(87 \%)\end{array}$ & $\begin{array}{l}34 / 45 \\
(76 \%)\end{array}$ & 0.055 & $\begin{array}{l}275 / 419 \\
(66 \%)\end{array}$ & $\begin{array}{l}20 / 33 \\
(61 \%)\end{array}$ & 0.56 \\
\hline $\begin{array}{l}\text { Drinking habit } ₫ \text { the } \\
\text { preceding year》 }\end{array}$ & $\begin{array}{l}429 / 710 \\
(60 \%)\end{array}$ & $\begin{array}{l}166 / 213 \\
(78 \%)\end{array}$ & $\begin{array}{l}30 / 45 \\
(67 \%)\end{array}$ & 0.11 & $\begin{array}{l}215 / 419 \\
(51 \%)\end{array}$ & $\begin{array}{l}18 / 33 \\
(55 \%)\end{array}$ & 0.72 \\
\hline $\begin{array}{l}\text { Unchanged } \\
\text { drinking habit }\end{array}$ & $\begin{array}{l}159 / 423 \\
(38 \%)\end{array}$ & $\begin{array}{l}69 / 167 \\
(41 \%)\end{array}$ & $\begin{array}{l}11 / 29 \\
(38 \%)\end{array}$ & 0.73 & $\begin{array}{l}77 / 209 \\
(37 \%)\end{array}$ & $\begin{array}{l}2 / 18 \\
(11 \%)\end{array}$ & 0.052 \\
\hline $\begin{array}{l}\text { Improved drinking } \\
\text { habit }\end{array}$ & $\begin{array}{l}264 / 423 \\
(62 \%)\end{array}$ & $\begin{array}{l}98 / 167 \\
(59 \%)\end{array}$ & $\begin{array}{l}18 / 29 \\
(62 \%)\end{array}$ & 0.73 & $\begin{array}{l}132 / 209 \\
(63 \%)\end{array}$ & $\begin{array}{l}16 / 18 \\
(89 \%)\end{array}$ & 0.052 \\
\hline AUDIT score & $2(0-5)$ & $4(1-9)$ & $\begin{array}{l}6(0- \\
12)\end{array}$ & 0.72 & $0(0-3)$ & $1(0-2)$ & 0.80 \\
\hline $\begin{array}{l}\text { AUDIT score of } 8 \\
\text { or more }\end{array}$ & $\begin{array}{l}111 / 666 \\
(17 \%)\end{array}$ & $\begin{array}{l}61 / 195 \\
(31 \%)\end{array}$ & $\begin{array}{l}17 / 39 \\
(44 \%)\end{array}$ & 0.14 & $\begin{array}{l}32 / 401 \\
(8.0 \%)\end{array}$ & $\begin{array}{l}1 / 31 \\
(3.2 \%)\end{array}$ & 0.54 \\
\hline Smoking $\rrbracket$ lifetime $\rrbracket$ & $\begin{array}{l}265 / 693 \\
(38 \%)\end{array}$ & $\begin{array}{l}129 / 209 \\
(62 \%)\end{array}$ & $\begin{array}{l}32 / 44 \\
(73 \%)\end{array}$ & 0.17 & $\begin{array}{l}96 / 407 \\
(24 \%)\end{array}$ & $\begin{array}{l}6 / 33 \\
(18 \%)\end{array}$ & 0.48 \\
\hline \multicolumn{8}{|l|}{ (continued). } \\
\hline & All (721) & \multicolumn{3}{|l|}{ Male (260) } & \multicolumn{3}{|l|}{ Female (461) } \\
\hline & & \multicolumn{3}{|c|}{$\begin{array}{l}\text { Postoperative } \\
\text { complications }\end{array}$} & \multicolumn{3}{|l|}{$\begin{array}{l}\text { Postoperative } \\
\text { complications }\end{array}$} \\
\hline & & No (215) & $\begin{array}{l}\text { Yes } \\
(45)\end{array}$ & $p$ value & No (427) & $\begin{array}{l}\text { Yes } \\
(34)\end{array}$ & $\mathrm{p}$ value \\
\hline
\end{tabular}




\begin{tabular}{llllllll}
$\begin{array}{l}\text { Smoking habit (the } \\
\text { preceding year) }\end{array}$ & $\begin{array}{l}144 / 693 \\
(21 \%)\end{array}$ & $\begin{array}{l}70 / 209 \\
(33 \%)\end{array}$ & $\begin{array}{l}17 / 44 \\
(39 \%)\end{array}$ & 0.51 & $\begin{array}{l}54 / 407 \\
(13 \%)\end{array}$ & $\begin{array}{l}3 / 33 \\
(9.1 \%)\end{array}$ & 0.68 \\
\hline $\begin{array}{l}\text { Unchanged } \\
\text { smoking habit }\end{array}$ & $\begin{array}{l}41 / 140 \\
(29 \%)\end{array}$ & $\begin{array}{l}22 / 69 \\
(32 \%)\end{array}$ & $\begin{array}{l}3 / 16 \\
(19 \%)\end{array}$ & 0.46 & $\begin{array}{l}16 / 53 \\
(30 \%)\end{array}$ & $0 / 2$ & 0.89 \\
\hline $\begin{array}{l}\text { Improved smoking } \\
\text { habit }\end{array}$ & $\begin{array}{l}99 / 140 \\
(71 \%)\end{array}$ & $\begin{array}{l}47 / 69 \\
(68 \%)\end{array}$ & $\begin{array}{l}13 / 16 \\
(81 \%)\end{array}$ & 0.46 & $\begin{array}{l}37 / 53 \\
(70 \%)\end{array}$ & $2 / 2$ & 0.89 \\
\hline FTND score & $0(0-0)$ & $0(0-2)$ & $0(0-3)$ & 0.57 & $0(0-0)$ & $0(0-0)$ & 0.49 \\
\hline $\begin{array}{l}\text { FTND score of 3 or } \\
\text { more }\end{array}$ & $\begin{array}{l}92 / 686 \\
(13 \%)\end{array}$ & $\begin{array}{l}48 / 207 \\
(23 \%)\end{array}$ & $\begin{array}{l}11 / 43 \\
(26 \%)\end{array}$ & 0.74 & $32 / 404$ & $1 / 32$ & 0.52 \\
\hline K6 score & $3(1-6)$ & $2(0-5)$ & $3(1-5)$ & 0.68 & $3(1-6)$ & $5(2-8)$ & 0.12 \\
\hline $\begin{array}{l}\text { K6 score of } 8 \text { or } \\
\text { more }\end{array}$ & $\begin{array}{l}108 / 694 \\
(16 \%)\end{array}$ & $\begin{array}{l}25 / 210 \\
(12 \%)\end{array}$ & $\begin{array}{l}4 / 45 \\
(8.9 \%)\end{array}$ & 0.75 & $\begin{array}{l}69 / 405 \\
(17 \%)\end{array}$ & $\begin{array}{l}10 / 34 \\
(29 \%)\end{array}$ & 0.071 \\
\hline HADS score & $8(4-13)$ & $8(4-12)$ & $\begin{array}{l}8(4- \\
13)\end{array}$ & 0.49 & $8(5-13)$ & $8(5-$ & 0.51 \\
\hline HADS score of 20 & $\begin{array}{l}40 / 690 \\
\text { or more }\end{array}$ & $\begin{array}{l}9 / 207 \\
(4.3 \%)\end{array}$ & $\begin{array}{l}3 / 45 \\
(6.7 \%)\end{array}$ & 0.78 & $24 / 404$ & $\begin{array}{l}4 / 34 \\
(12 \%)\end{array}$ & 0.33
\end{tabular}

Categorical variables are shown as proportion (\%), and continuous variables are shown as median (interquartile range).

BMI, body mass index; ASA-PS, the American Society of Anesthesiologists physical status classification; sAs, Surgical Apgar score; AUDIT, Alcohol Use Disorders Identification Test; FTND, Fagerström Test for Nicotine Dependence; K6, 6-item Kessler Psychological Distress Scale; HADS, Hospital Anxiety and Depression Scale ${ }^{*} \mathrm{p}<0.05$ 
Table 3. Sex- and age-adjusted analysis to examine the association of postoperative complications and patient characteristics

\begin{tabular}{|c|c|c|c|c|c|}
\hline & \multicolumn{2}{|c|}{$\begin{array}{l}\text { Postoperative } \\
\text { complications }\end{array}$} & \multicolumn{3}{|l|}{$\begin{array}{l}\text { M-W U/ } x^{2} \\
\text { test }\end{array}$} \\
\hline & No (642) & Yes (79) & $p$ value & OR $(95 \% \mathrm{Cl})$ & $p$ value \\
\hline BMI $\left(\mathrm{kg} / \mathrm{m}^{2}\right)$ & $23(21-25)$ & $23(21-25)$ & 0.91 & $0.96(0.89-1.03)$ & 0.26 \\
\hline ASA-PS I & 202 & 10 & $0.002^{\star}$ & $\begin{array}{l}\text { II/I } 1.89(0.93- \\
4.15)\end{array}$ & 0.077 \\
\hline ASA-PS II & 417 & 66 & & $\begin{array}{l}\text { III/I } 1.37(0.28- \\
5.18)\end{array}$ & 0.67 \\
\hline ASA-PS III & 23 & 3 & & $\begin{array}{l}\text { III/II } 0.73(0.17- \\
2.21)\end{array}$ & 0.61 \\
\hline $\begin{array}{l}\text { Preoperative use of } \\
\text { psychotropics }\end{array}$ & $\begin{array}{l}74 / 615 \\
(12 \%)\end{array}$ & $\begin{array}{l}11 / 78 \\
(14 \%)\end{array}$ & 0.58 & $0.95(0.47-1.93)$ & 0.89 \\
\hline $\begin{array}{l}\text { Anesthesia time (minutes. hours } \\
\text { in OR) }\end{array}$ & $\begin{array}{l}169(127- \\
234)\end{array}$ & $\begin{array}{l}298(210- \\
389)\end{array}$ & $p<0.001^{\star}$ & $1.53(1.37-1.73)$ & $p<0.001^{*}$ \\
\hline sAs & $8(7-9)$ & $7(6-8)$ & $0.001 *$ & $0.82(0.70-0.97)$ & $0.019 *$ \\
\hline Drinking $\mathbb{\| l i f e t i m e \rrbracket}$ & $\begin{array}{l}460 / 632 \\
(73 \%)\end{array}$ & $\begin{array}{l}54 / 78 \\
(69 \%)\end{array}$ & 0.51 & $0.79(0.46-1.41)$ & 0.42 \\
\hline 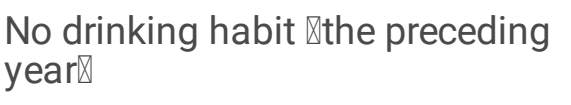 & $\begin{array}{l}249 / 632 \\
(39 \%)\end{array}$ & $\begin{array}{l}30 / 78 \\
(38 \%)\end{array}$ & 0.87 & $0.99(0.58-1.69)$ & 0.98 \\
\hline Unchanged drinking habit & $\begin{array}{l}146 / 376 \\
(39 \%)\end{array}$ & $\begin{array}{l}13 / 47 \\
(28 \%)\end{array}$ & 0.14 & $0.74(0.34-1.53)$ & 0.42 \\
\hline Improved drinking habit & $\begin{array}{l}230 / 376 \\
(61 \%)\end{array}$ & $\begin{array}{l}34 / 47 \\
(72 \%)\end{array}$ & 0.14 & $0.74(0.69-2.20)$ & 0.47 \\
\hline AUDIT score & $1(0-5)$ & $2(0-8)$ & 0.13 & $1.23(0.97-1.07)$ & 0.45 \\
\hline AUDIT score of 8 or more & $\begin{array}{l}93 / 596 \\
(16 \%)\end{array}$ & $\begin{array}{l}18 / 77 \\
(23 \%)\end{array}$ & 0.084 & $1.48(0.77-2.76)$ & 0.24 \\
\hline 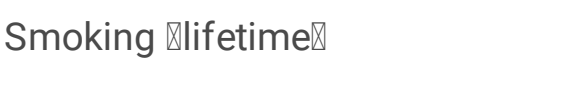 & $\begin{array}{l}225 / 616 \\
(37 \%)\end{array}$ & $\begin{array}{l}38 / 77 \\
(49 \%)\end{array}$ & $0.029 *$ & $1.39(0.80-2.39)$ & 0.24 \\
\hline
\end{tabular}

(continued).

\begin{tabular}{|c|c|c|c|c|c|}
\hline & \multicolumn{2}{|c|}{$\begin{array}{l}\text { Postoperative } \\
\text { complications }\end{array}$} & \multicolumn{2}{|c|}{$\begin{array}{l}\text { M-W U/ } \mathrm{x}^{2} \\
\text { test }\end{array}$} & \multirow[b]{2}{*}{$p$ value } \\
\hline & No (642) & Yes (79) & $p$ value & OR $(95 \% \mathrm{Cl})$ & \\
\hline $\begin{array}{l}\text { No smoking habit } \triangle \text { the } \\
\text { preceding year》 }\end{array}$ & $\begin{array}{l}492 / 616 \\
(80 \%)\end{array}$ & $\begin{array}{l}57 / 77 \\
(74 \%)\end{array}$ & 0.23 & $0.68(0.38-1.27)$ & 0.22 \\
\hline Unchanged smoking habit & $\begin{array}{l}38 / 122 \\
(31 \%)\end{array}$ & $3 / 18(17 \%)$ & 0.34 & $0.79(0.18-2.46)$ & 0.71 \\
\hline
\end{tabular}




\begin{tabular}{llllll} 
Improved smoking habit & $\begin{array}{l}84 / 122 \\
(69 \%)\end{array}$ & $\begin{array}{l}15 / 18 \\
(83 \%)\end{array}$ & 0.34 & $1.48(0.74-2.83)$ & 0.26 \\
\hline FTND score & $0(0-0)$ & $0(0-0)$ & 0.44 & $1.10(0.96-1.25)$ & 0.15 \\
\hline FTND score of 3 or more & $\begin{array}{l}80 / 611 \\
(13 \%)\end{array}$ & $\begin{array}{l}12 / 75 \\
(16 \%)\end{array}$ & 0.49 & $0.79(0.40-1.68)$ & 0.53 \\
\hline K6 score & $3(1-6)$ & $4(1-6)$ & 0.55 & $1.07(1.00-113)$ & $0.040^{\star}$ \\
\hline K6 score of 8 or more & $\begin{array}{l}94 / 615 \\
(15 \%)\end{array}$ & $\begin{array}{l}14 / 79 \\
(18 \%)\end{array}$ & 0.57 & $1.51(0.77-2.80)$ & 0.22 \\
\hline HADS score & $8(4-13)$ & $8(5-14)$ & 0.96 & $1.02(0.98-1.06)$ & 0.24 \\
\hline HADS score of 20 or more & $\begin{array}{l}33 / 611 \\
(5.5 \%)\end{array}$ & $\begin{array}{l}7 / 79 \\
(8.9 \%)\end{array}$ & 0.22 & $1.97(0.76-4.52)$ & 0.15 \\
\hline Length of stay after surgery & $8(5-12)$ & $21(12-38)$ & $p<0.001^{*}$ & & \\
\hline
\end{tabular}

Categorical variables are shown as proportion (\%), and continuous variables are shown as median (interquartile range).

M-W U, Mann-Whitney U test; $x^{2}$ test, chi-squared test; OR, odds ratio; $\mathrm{Cl}$, confidence interval; BMI, body mass index; ASA-PS, the American Society of Anesthesiologists physical status classification; sAs, Surgical Apgar score; AUDIT, Alcohol Use Disorders Identification Test; FTND, Fagerström Test for Nicotine Dependence; K6, 6item Kessler Psychological Distress Scale; HADS, Hospital Anxiety and Depression Scale

${ }^{*} \mathrm{p}<0.05$

We applied multivariable analysis to examine factors associated with postoperative complications. The correlation between anesthesia time and surgical time, and between the K6 and HADS score, were both 0.6 and higher. As such, only the more highly causal variable from each pair was included in the final model (Model 1-4) (anesthesia time and K6 score, respectively). The significant risk factors for the development of postoperative complications in each model were as follows (Table 4): Model 1: male sex (OR: 1.86 [Cl: 1.00-3.49], p =0.049), anesthesia time (hours) (OR: 1.55 [Cl: 1.35-1.80], p < 0.001), and K6 score (OR: 1.08 [Cl: 1.00-1.16], p = 0.033); Model 2: old age (for every 10 years) (OR: 1.34 [Cl: 1.05-1.74], $p=0.020$ ), anesthesia time (hours) (OR: 1.51 [Cl: 1.32-1.75], $p<0.001$ ), and K6 score (OR: 1.08 [Cl: 1.00-1.15], $p=0.041$ ); Model 3 (male): anesthesia time (hours) (OR: 1.47 [Cl: 1.23-1.77], $\mathrm{p}<0.001$ ); Model 4 (female): anesthesia time (hours) (OR: 1.62 [Cl: 1.30-2.06], $\mathrm{p}<$ 0.001 ) and K6 score (OR: 1.12 [Cl: 1.02-1.23], $p=0.017)$. Improved drinking/smoking habits and cessation of drinking/smoking after surgical decision-making were not associated with postoperative complications. 
Table 4. Factors associated with postoperative complications (Adjusted multivariable analysis)

\begin{tabular}{|c|c|c|c|c|c|c|c|c|}
\hline & \multicolumn{2}{|l|}{ Model 1} & \multicolumn{2}{|l|}{ Model 2} & \multicolumn{2}{|c|}{ Model 3 (male) } & \multicolumn{2}{|c|}{ Model 4 (female) } \\
\hline & $\begin{array}{l}\text { OR }(95 \% \\
\mathrm{Cl})\end{array}$ & $\mathrm{p}$ value & $\begin{array}{l}\text { OR }(95 \% \\
\mathrm{Cl})\end{array}$ & $p$ value & $\begin{array}{l}\text { OR } \\
(95 \% \\
\text { Cl) }\end{array}$ & $p$ value & $\begin{array}{l}\text { OR } \\
(95 \% \\
\text { Cl) }\end{array}$ & $\mathrm{p}$ value \\
\hline Sex (male) & $\begin{array}{l}1.86 \\
(1.00- \\
3.49)\end{array}$ & $0.049 *$ & $\begin{array}{l}1.63 \\
(0.85- \\
3.13)\end{array}$ & 0.14 & & & & \\
\hline Age/10 & $\begin{array}{l}1.27 \\
(0.99- \\
1.64)\end{array}$ & 0.054 & $\begin{array}{l}1.34 \\
(1.05- \\
1.74)\end{array}$ & $0.020 *$ & $\begin{array}{l}1.30 \\
(0.91- \\
1.92)\end{array}$ & 0.15 & $\begin{array}{l}1.35 \\
(0.92- \\
2.02)\end{array}$ & 0.12 \\
\hline $\mathrm{BMI}\left(\mathrm{kg} / \mathrm{m}^{2}\right)$ & $\begin{array}{l}0.96 \\
(0.89- \\
1.04)\end{array}$ & 0.37 & $\begin{array}{l}0.99 \\
(0.91- \\
1.07)\end{array}$ & 0.75 & $\begin{array}{l}1.03 \\
(0.91- \\
1.16)\end{array}$ & 0.66 & $\begin{array}{l}0.93 \\
(0.81- \\
1.04)\end{array}$ & 0.21 \\
\hline ASA-PS $\|^{\dagger}$ & $\begin{array}{l}1.55 \\
(0.72- \\
3.58)\end{array}$ & 0.27 & $\begin{array}{l}1.35 \\
(0.62- \\
3.13)\end{array}$ & 0.46 & $\begin{array}{l}1.78 \\
(0.45- \\
11.8)\end{array}$ & 0.45 & $\begin{array}{l}1.49 \\
(0.54- \\
4.28)\end{array}$ & 0.45 \\
\hline ASA-PS III ${ }^{\dagger}$ & $\begin{array}{l}1.20 \\
(0.22- \\
5.10)\end{array}$ & 0.82 & $\begin{array}{l}0.75 \\
(0.10- \\
3.60)\end{array}$ & 0.74 & $\begin{array}{l}0.70 \\
(0.03- \\
9.07)\end{array}$ & 0.79 & $\begin{array}{l}1.26 \\
(0.06- \\
10.5)\end{array}$ & 0.85 \\
\hline sAs & $\begin{array}{l}1.10 \\
(0.90- \\
1.38)\end{array}$ & 0.37 & $\begin{array}{l}1.09 \\
(0.88- \\
1.37)\end{array}$ & 0.44 & $\begin{array}{l}1.08 \\
(0.81- \\
1.47)\end{array}$ & 0.62 & $\begin{array}{l}1.10 \\
(0.81- \\
1.57)\end{array}$ & 0.56 \\
\hline $\begin{array}{l}\text { Anesthesia time } \\
\text { (hours) }\end{array}$ & $\begin{array}{l}1.55 \\
(1.35- \\
1.80)\end{array}$ & $p<0.001^{*}$ & $\begin{array}{l}1.51 \\
(1.32- \\
1.75)\end{array}$ & $p<0.001^{*}$ & $\begin{array}{l}1.47 \\
(1.23- \\
1.77)\end{array}$ & $p<0.001 *$ & $\begin{array}{l}1.62 \\
(1.30- \\
2.06)\end{array}$ & $p<0.001^{*}$ \\
\hline $\begin{array}{l}\text { Unchanged } \\
\text { drinking habit }\end{array}$ & $\begin{array}{l}0.93 \\
(0.41- \\
2.07)\end{array}$ & 0.87 & & & & & & \\
\hline $\begin{array}{l}\text { Improved } \\
\text { drinking habit }{ }^{\ddagger}\end{array}$ & $\begin{array}{l}0.87 \\
(1.45- \\
1.66)\end{array}$ & 0.66 & & & & & & \\
\hline $\begin{array}{l}\text { Unchanged } \\
\text { smoking habit } ¥\end{array}$ & $\begin{array}{l}1.51 \\
(0.32- \\
5.18)\end{array}$ & 0.56 & & & & & & \\
\hline $\begin{array}{l}\text { Improved } \\
\text { smoking habit }\end{array}$ & $\begin{array}{l}0.91 \\
(0.41- \\
1.95)\end{array}$ & 0.82 & & & & & & \\
\hline K6 score & $\begin{array}{l}1.08 \\
(1.00- \\
1.16)\end{array}$ & $0.033^{\star}$ & $\begin{array}{l}1.08 \\
(1.00- \\
1.15)\end{array}$ & $0.041 *$ & $\begin{array}{l}1.04 \\
(0.93- \\
1.16)\end{array}$ & 0.52 & $\begin{array}{l}1.12 \\
(1.02- \\
1.23)\end{array}$ & $0.017 *$ \\
\hline AUDIT score & & & $\begin{array}{l}1.00 \\
(0.95- \\
1.06)\end{array}$ & 0.88 & $\begin{array}{l}1.00 \\
(0.94- \\
1.06)\end{array}$ & 0.99 & $\begin{array}{l}1.02 \\
(0.88- \\
1.13)\end{array}$ & 0.76 \\
\hline FTND score & & & $\begin{array}{l}1.08 \\
(0.92- \\
1.25)\end{array}$ & 0.31 & $\begin{array}{l}1.12 \\
(0.95- \\
1.31)\end{array}$ & 0.18 & $\begin{array}{l}0.73 \\
(0.37- \\
1.16)\end{array}$ & 0.21 \\
\hline
\end{tabular}


(continued).

\begin{tabular}{|lllll}
\hline AUC & $81 \%$ & $80 \%$ & $79 \%$ & $78 \%$ \\
\hline Accuracy & $89 \%$ & $89 \%$ & $83 \%$ & $93 \%$
\end{tabular}

OR, odds ratio; $\mathrm{Cl}$, confidence interval; BMI, body mass index; ASA-PS, the American Society of Anesthesiologists physical status classification; sAs, Surgical

Apgar score; K6, 6-item Kessler Psychological Distress Scale; AUDIT, Alcohol Use Disorders Identification Test; FTND, Fagerström Test for Nicotine Dependence; AUC, area under the curve

${ }^{\dagger}$ Odds ratio of ASA-PS I

‡Odds ratio of "no drinking/smoking habits for the preceding year leading up to surgical decision-making" ${ }^{\star} \mathrm{p}<0.05$

\section{Discussion}

This study found that preoperative psychological stress is an independent risk factor for postoperative complications in adult surgical patients. In addition, K6 may be a useful tool for its evaluation.

First, preoperative psychological stress predicted the subsequent occurrence of postoperative complications. This may suggest that being optimistic for the surgery had positive effect on faster recovery, and preoperative intervention against psychological stress may reduce the risk of postoperative complications. In previous studies, preoperative psychological stress or depression has been reported to be associated with postoperative complications and poorer recovery $[8,26]$. For example, depression increases postoperative wound infection rates due to decreased immunity $[27,28]$. Furthermore, when depression and pain occur simultaneously, depression lowers the pain threshold and worsens both conditions $[29,30]$. Pain is an obstacle to early postoperative recovery as it delays patient mobilization, causes an increase in blood pressure, and leads to infections, hemorrhage, and delirium [31]. Since correlations between depression and postoperative complications have been previously reported, psychological stress, which is a precursor to depression, can also cause immunosuppression and aggravation of pain that trigger postoperative complications [32]; thus, perioperative psychological stress may adversely affect postoperative outcomes.

In this study, the $\mathrm{K} 6$ was shown to be a convenient and effective tool for evaluating psychological stress. Although no study has yet directly compared the HADS to the K6 or evaluated the association between the K6 score and risks of postoperative complications, the $\mathrm{K} 6$ may be useful for simple screening of preoperative psychological stress as elderly patients can also complete in the limited time available on the day before surgery.

Unlike previous studies, our results showed that improved drinking/smoking habits, the AUDIT score (alcohol dependence), and the FTND score (nicotine dependence) were not associated with postoperative complications in multivariable analysis. Assuming that preoperative psychological stress is a risk factor for postoperative complications, this suggests that drinking/smoking cessation can itself be hugely stressful to mental health [33], which may have counteracted any positive results of cessation, such as improving immune function and cardiopulmonary function [34-36]. Additionally, since women made up 64\% of all cases in this study, the 
proportion of patients who smoked within the year leading up to the surgical decision was low (21\%), which may explain the discrepancies with previous studies. In fact, mean FTND scores of 3.9 points in Canadian patients [37] and 4.1 points in Chinese patients have been reported in past studies [38]. Regarding AUDIT scores, 6.5 points (median) have been reported in American intensive care unit patients [39], and 7.9 points (mean) were reported in Japanese firefighters [15]. In comparison, the patient group in the present study had lower FTND scores (mean 0.7, median 0 ) and AUDIT (mean 3.6, median 2). The growing importance of health literacy in surgical populations and smoking/drinking cessation instructions from surgeons also likely contributed to the decline of the influence of smoking and drinking [40]. Moreover, another reason may be that $29 \%$ of all surgeries in this study were breast surgery, which has a low rate of postoperative complications, regardless of drinking/smoking habits. However, several studies have reported the long-term benefits of smoking cessation due to surgical decision-making. In this study, the proportion of patients who either reduced or quit their drinking/smoking habits between surgical decision-making and surgery were $62 \%$ and $71 \%$, respectively. These results support the belief that surgical decision-making, as a life event, is a highly effective "teachable moment" for lifetime smoking cessation and longterm moderation of drinking habits [41, 42].

Modifiable risk factors were anesthesia (surgical) time and the K6 score (psychological stress) in this study. Therefore, shortening the surgical time is important to reduce postoperative complications, particularly in elderly patients. Psychological support is an emerging area of prehabilitation practice [43]. Although HADS is used, an optimal preoperative screening tool is yet to emerge [4]; K6 may be simpler and more useful for preoperative screening of mental status.

This study had some limitations. First, since this was an observational study, we cannot make any inferences concerning the causal association between psychological stress and postoperative complications. Significant psychological stress observed in preoperative patients may simply be a progression of depressive tendencies that occur as a result of disease progression and announcement of their illness. It is possible that the patient's condition, which was not assessed by ASA-PS, was already at risk of developing postoperative complications. Second, it is also likely that the number of missing items and recall bias, which are typical issues when using questionnaires, are higher than normal in our hospital given that we treat many elderly patients. Especially in elderly cancer patients, cognitive function is likely to be impaired by chemotherapy, electrolyte and endocrine abnormalities, brain tumors or brain metastases, and malnutrition [44]; therefore, the questions in this survey may have been too many. Hence, at most diagnosis procedure combination hospitals where patients are admitted one day before surgery, it may be necessary to shorten the questionnaire by limiting items to those that have the most significant effects on patient outcomes. Third, the effect of recent history of anesthesia and surgery on postoperative outcomes was not included in the analysis. This could affect preoperative mental status; however, we did not ask this question because many patients often misinterpret sedation during neuraxial anesthesia and examinations as general anesthesia with endotracheal intubation. We consider that we were able to assess the impact of surgical experience on preoperative psychological distress to some extent in $\mathrm{K} 6$.

In conclusion, our analysis suggests that psychological stress, as evaluated by the K6, may increase the frequency of postoperative complications. Reports on the use of the $\mathrm{K} 6$ in the perioperative period have thus far been limited. Future studies should consider $\mathrm{K} 6$ while assessing the need for preoperative psychological support or intervention.

\section{Abbreviations}


$\mathrm{AE}$, adverse event; ASA-PS, the American Society of Anesthesiologists physical status classification; AUC, area under the curve; AUDIT, Alcohol Use Disorders Identification Test; BMI, body mass index; Cl, confidence interval; FTND, Fagerström Test for Nicotine Dependence; HADS, Hospital Anxiety and Depression Scale; JCOG, Japan Clinical Oncology Group; K6, 6-item Kessler Psychological Distress Scale; OR, odds ratio; sAs, Surgical Apgar score

\section{Declarations}

\section{Ethics approval and consent to participate}

This study was approved by the Ethics Committee of the Kitakyushu Municipal Medical Center and Tottori University Hospital. The authors declare that the all recruited patients provided completed and signed questionnaires to attending anesthesiologists, implying informed consent for the study, and that this work was conducted in accordance with the Declaration of Helsinki (1964) and its subsequent amendments.

\section{Consent for publication}

Informed consent for publication of clinical research was obtained from all patients.

\section{Availability of data and materials}

Data from the reported cases will be made available on reasonable request from the corresponding author.

\section{Competing interests}

The authors declare no competing interests.

\section{Funding}

This study received no funding from any private or government agency.

\section{Author contributions}

YM conceived the study, researched the literature, and wrote the manuscript. YM and HM contributed to management and supervision of anesthesia. YO contributed to reviewing/editing of the draft and statistical analysis. All authors read and approved the final manuscript.

\section{Acknowledgements}

We would like to thank Editage for English language editing. 


\section{References}

1. International Surgical Outcomes Study group Global patient outcomes after elective surgery: prospective cohort study in 27 low-, middle- and high-income countries. Br J Anaesth. 2016;117:601-9.

2. Pearse RM, Moreno RP, Bauer P, Pelosi P, Metnitz P, Spies C, et al. Mortality after surgery in Europe: a 7 day cohort study. Lancet. 2012;380:1059-65.

3. Schwab M, Brindl N, Studier-Fischer A, Tu T, Gsenger J, Pilgrim M, et al. Postoperative complications and mobilisation following major abdominal surgery with vs. without fitness tracker-based feedback (EXPELLIARMUS): study protocol for a student-led multicentre randomised controlled trial (CHIR-Net SIGMA study group). Trials. 2020; doi:10.1186/s13063-020-4220-8.

4. Durrand J, Singh SJ, Danjoux G. Prehabilitation. Clin Med (Lond). 2019;19:458-64.

5. Eliasen M, Grønkjær M, Skov-Ettrup LS, Mikkelsen SS, Becker U, Tolstrup JS, et al. Preoperative alcohol consumption and postoperative complications: a systematic review and meta-analysis. Ann Surg. 2013;258:930-42.

6. Grønkjær M, Eliasen M, Skov-Ettrup LS, Tolstrup JS, Christiansen AH, Mikkelsen SS, et al. Preoperative smoking status and postoperative complications: a systematic review and meta-analysis. Ann Surg. 2014;259:52-71.

7. Turan A, Mascha EJ, Roberman D, Turner PL, You J, Kurz A, et al. Smoking and perioperative outcomes. Anesthesiology. 2011;114:837-46.

8. Ghoneim MM, O’Hara MW. Depression and postoperative complications: an overview. BMC Surg. 2016; doi:10.1186/s12893-016-0120-y.

9. Thomas G, Tahir MR, Bongers BC, Kallen VL, Slooter GD, van Meeteren NL. Prehabilitation before major intraabdominal cancer surgery: A systematic review of randomised controlled trials. Eur $\mathrm{J}$ Anaesthesiol. 2019;36:933-45.

10. McDonald S, Yates D, Durrand JW, Kothmann E, Sniehotta FF, Habgood A, et al. Exploring patient attitudes to behaviour change before surgery to reduce peri-operative risk: preferences for short- vs. long-term behaviour change. Anaesthesia. 2019;74:1580-8.

11. Levett DZ, Edwards M, Grocott M, Mythen M. Preparing the patient for surgery to improve outcomes. Best Pract Res Clin Anaesthesiol. 2016;30:145-57.

12. Hosoda T, Osaki Y, Okamoto H, Wada T, Otani S, Mu H, et al. Evaluation of relationships among occupational stress, alcohol dependence and other factors in male personnel in a Japanese local fire fighting organization. Yonago Acta Med. 2012;55:63-8.

13. Heatherton TF, Kozlowski LT, Frecker RC, Fagerström KO. The Fagerström test for nicotine dependence: a revision of the Fagerström Tolerance Questionnaire. Br J Addict. 1991;86:1119-27.

14. Nakamura K, Sakurai M, Miura K, Morikawa Y, Nagasawa SY, Ishizaki M, et al. Nicotine dependence and costeffectiveness of individualized support for smoking cessation: evidence from practice at a worksite in Japan. PloS One. 2013; doi:10.1371/journal.pone.0055836.

15. Zigmond AS, Snaith RP. The hospital anxiety and depression scale. Acta Psychiatr Scand. 1983;67:361-70.

16. Kugaya A, Akechi T, Okuyama T, Okamura H, Uchitomi Y. Screening for psychological distress in Japanese cancer patients. Jpn J Clin Oncol. 1998;28:333-8. 
17. Kessler RC, Andrews G, Colpe LJ, Hiripi E, Mroczek DK, Normand SLT, et al. Short screening scales to monitor population prevalences and trends in non-specific psychological distress. Psychol Med. 2002;32:959-76.

18. Arnaud B, Malet L, Teissedre F, Izaute M, Moustafa F, Geneste J, et al. Validity study of Kessler's psychological distress scales conducted among patients admitted to French emergency department for alcohol consumption-related disorders. Alcohol Clin Exp Res. 2010;34:1235-45.

19. Vigod SN, Kurdyak PA, Stewart DE, Gnam WH, Goering PN. Depressive symptoms as a determinant of breast and cervical cancer screening in women: a population-based study in Ontario, Canada. Arch Womens Ment Health. 2011;14:159-68.

20. Phan K, Kim JS, Lee NJ, Kothari P, Cho SK. Relationship between ASA scores and 30-day readmissions in patients undergoing anterior cervical discectomy and fusion. Spine. 2017;42:85-91.

21. Owens WD, Felts JA, Spitznagel EL. ASA physical status classifications: a study of consistency of ratings. Anesthesiology. 1978;49:239-43.

22. Gawande AA, Kwaan MR, Regenbogen SE, Lipsitz SA, Zinner MJ. An Apgar score for surgery. J Am Coll Surg. 2007;204:201-8.

23. Regenbogen SE, Ehrenfeld JM, Lipsitz SR, Greenberg CC, Hutter MM, Gawande AA. Utility of the surgical Apgar score: validation in 4119 patients. Arch Surg. 2009;144:30-6.

24. Dindo D, Demartines N, Clavien PA. Classification of surgical complications: a new proposal with evaluation in a cohort of 6336 patients and results of a survey. Ann Surg. 2004;240:205-13.

25. Katayama H, Kurokawa Y, Nakamura K, Ito H, Kanemitsu Y, Masuda N, et al. Extended Clavien-Dindo classification of surgical complications: Japan Clinical Oncology Group postoperative complications criteria. Surg Today. 2016;46:668-85.

26. Nilsson U, Dahlberg K, Jaensson M. Low Preoperative Mental and Physical Health is Associated with Poorer Postoperative Recovery in Patients Undergoing Day Surgery: A Secondary Analysis from a Randomized Controlled Study. World J Surg. 2019;43:1949-56.

27. Theodore DA, Goodwin RD, Zhang YV, Schneider N, Gordon RJ. History of depression and increased risk of sternal wound infection after cardiothoracic surgery: a novel and potentially modifiable risk factor. Open Forum Infect Dis. 2019; doi:10.1093/ofid/ofz083.

28. Althoff AD, Reeves RA, Traven SA, Slone HS, Deal DN, Werner BC. Risk factors for infection following total wrist arthroplasty and arthrodesis: an analysis of 6641 patients. Hand. 2019; doi:10.1177/1558944719890036.

29. Rhudy JL, Meagher MW. Fear and anxiety: divergent effects on human pain thresholds. Pain. 2000;84:65-75.

30. Gureje O. Treating chronic pain in the context of comorbid depression. Pain. 2008;134:3-4.

31. van Boekel RLM, Warlé MC, Nielen RGC, Vissers KCP, van der Sande R, Bronkhorst EM, et al. Relationship between postoperative pain and overall 30-day complications in a broad surgical population: an observational study. Ann Surg. 2019;269:856-65.

32. Hammen C. Risk Factors for Depression: An Autobiographical Review. Annu Rev Clin Psychol. 2018;14:1-28.

33. Kim SJ, Chae W, Park WH, Park MH, Park EC, Jang SI. The impact of smoking cessation attempts on stress levels. BMC Public Health. 2019;19:267.

34. Sørensen LT. Wound healing and infection in surgery: the pathophysiological impact of smoking, smoking cessation, and nicotine replacement therapy: a systematic review. Ann Surg. 2012;255:1069-79. 
35. Sørensen LT. Wound healing and infection in surgery: the clinical impact of smoking and smoking cessation: a systematic review and meta-analysis. Arch Surg. 2012;147:373-83.

36. Lau A, von Dossow V, Sander M, MacGuill M, Lanzke N, Spies C. Alcohol use disorder and perioperative immune dysfunction. Anesth Analg. 2009;108:916-20.

37. Lee SM, Landry J, Jones PM, Buhrmann O, Morley-Forster P. The effectiveness of a perioperative smoking cessation program: a randomized clinical trial. Anesth Analg. 2013;117:605-13.

38. Yu C, Shi Y, Kadimpati S, Sheng Y, Jing J, Schroeder D, et al. Perioperative smoking behavior of Chinese surgical patients. Anesth Analg. 2013;116:1238-46.

39. Afshar M, Burnham EL, Joyce C, Clark BJ, Yong M, Gaydos J, et al. Cut-point levels of phosphatidylethanol to identify alcohol misuse in a mixed cohort including critically ill patients. Alcohol Clin Exp Res. 2017;41:174553.

40. Hayter RR, Hess AS. Health numeracy and relative risk comprehension in perioperative patients and physicians. Anesth Analg. 2020;131:579-85.

41. Shi Y, Warner DO. Surgery as a teachable moment for smoking cessation. Anesthesiology. 2010;112:102-7.

42. Duffy SA, Khan MJ, Ronis DL, Fowler KE, Gruber SB, Wolf GT, et al. Health behaviors of head and neck cancer patients the first year after diagnosis. Head Neck. 2008;30:93-102.

43. Levett DZH, Grimmett C. Psychological factors, prehabilitation and surgical outcomes: evidence and future directions. Anaesthesia. 2019;74:36-42.

44. Janelsins MC, Kesler SR, Ahles TA, Morrow GR. Prevalence, mechanisms, and management of cancer-related cognitive impairment. Int Rev Psychiatry. 2014;26:102-13. 